\title{
Discrete Gel'fand-Levitan and Marchenko matrix equations and layer stripping algorithms for the discrete two-dimensional Schrödinger equation inverse scattering problem with a nonlocal potential
}

\author{
Andrew E Yagle $\dagger$ \\ Department of Electrical Engineering and Computer Science, The University of Michigan, Ann \\ Arbor, MI 48109-2122, USA \\ Received 10 July 1996, in final form 28 August 1997

\begin{abstract}
We develop discrete counterparts to the Gel'fand-Levitan and Marchenko integral equations for the two-dimensional (2D) discrete inverse scattering problem in polar coordinates with a nonlocal potential. We also develop fast layer stripping algorithms that solve these systems of equations exactly. The significance of these results is: (1) they are the first numerical implementation of Newton's multidimensional inverse scattering theory; (2) they show that the result will almost always be a nonlocal potential, unless the data are 'miraculous'; (3) they show that layer stripping algorithms implement fast 'split' signal processing fast algorithms; (4) they link 2D discrete inverse scattering with 2D discrete random field linear least-squares estimation; and (5) they formulate and solve 2D discrete Schrödinger equation inverse scattering problems in polar coordinates.
\end{abstract}

\section{Introduction}

\subsection{History}

The inverse scattering problem for the multidimensional Schrödinger equation with a timeindependent, local, nonspherically symmetric potential has many obvious applications in geophysics and nondestructive testing. In a series of papers beginning in 1980, Newton [1-3] generalized the Gel'fand-Levitan-Marchenko (GLM) machinery for the one-dimensional (1D) problem to three dimensional (3D). These results were interpreted in $[4,5]$ and elsewhere. Cheney [6] developed analogous results for the 2D problem. All of these methods reconstruct the scattering potential of a Schrödinger equation from the far-field responses (scattering amplitude) to an impulsive plane wave (incident from any direction) by solving either a Gel'fand-Levitan or Marchenko integral equation, and then computing the gradient of the solution at the boundary of its support.

Although these results are quite elegant, there has never been a numerical implementation of them. There is at least one major problem: since the data (scattering amplitude) have more degrees of freedom than the potential, the problem is overdetermined, and unless a so-called 'miracle' occurs in the gradient step noted above it is not at all clear what has been accomplished. Some sense of what happens when the data are not 'miraculous' is needed to interpret the numerical results of these procedures.

† E-mail address: aey@eecs.umich.edu 
One way around this problem is to consider the Schrödinger equation inverse scattering problem with a nonlocal potential diagonal in the radius [19]. This problem has applications to distributed systems with varying nonzero response times, and to linear least-squares estimation of random fields on the surface of a sphere of noisy observations $[7,8]$. The significance of this problem here is that [7] suggests that the Newton multidimensional GLM machinery is actually solving a nonlocal potential problem, which reduces to the (desired) local potential only if the 'miracle' occurs, since the scattering amplitude and nonlocal potential have equal degrees of freedom. Generalized Marchenko and Gel'fandLevitan integral equations for the 3D nonlocal problem were first given in $[9,10]$, where the degrees-of-freedom issue was discussed. Both integral equations and layer stripping algorithms were given in $[7,8]$.

An alternative approach to multidimensional inverse scattering is layer stripping. Layer stripping in 1D goes back to the Schur algorithm of 1917 and the dynamic deconvolution algorithms of the 1950s. A unified treatment connecting these algorithms to the 1D GLM machinery and (significantly) to the classical Levinson and fast Cholesky fast signal processing algorithms is given in $[11,12]$. Multidimensional layer stripping algorithms were developed in [13] and numerically implemented in [14], in which we found these algorithms to be surprisingly robust to varying discretization and noise levels. These results were explained in [15], in which a properly implemented multidimensional layer stripping algorithm was shown to be equivalent to a multichannel Levinson or Schur fast signal processing algorithm, and in which a feasibility condition on backscattered data was obtained, satisfaction of which implied that the layer stripping algorithm was stable. This shows the significance of relating inverse scattering algorithms to classical signal processing fast algorithms.

\subsection{Contributions of this paper}

In this paper, as in [15], we consider an entirely discrete Schrödinger equation inverse scattering problem. We consider the 2D problem for the convenience of notation, although the results extend to higher dimensions. These results differ from [15] in the following ways: (1) polar coordinates are used; (2) nonlocal potentials are used; and (3) far-field scattering data are allowed. The goal of this paper is to develop a discrete counterpart to the Newton GLM machinery, consisting of discrete systems of equations (instead of integral equations) and fast signal processing algorithms (instead of differential layer stripping algorithms) that solve exactly an explicitly discrete (as opposed to discretized) 2D Schrödinger equation inverse scattering problem.

The significance of these results is as follows.

(1) They constitute the first numerical implementation of Newton's multidimensional inverse scattering machinery, since the algorithms can be computer-implemented as they are, without any discretization or approximation.

(2) They show that numerical implementation of the Newton GLM machinery will almost always result in a nonlocal potential, unless 'miraculous' data are supplied. More precisely, if the data are miraculous, these discrete algorithms implement a discretization of Newton's GLM machinery. If not, then the result of applying a discretization of Newton's GLM machinery can be viewed as a discretized nonlocal potential. The mathematical significance of this to the continuous problem is unclear.

(3) They show that layer stripping algorithms implement fast signal processing algorithms, in this case the generalized split algorithms of [17]. This shows that these algorithms are numerically stable. 
(4) They explicitly link the discrete 2D inverse scattering problem with the $2 \mathrm{D}$ random field linear least-squares estimation problem, extending [16] to the discrete case.

(5) They formulate and solve the 2D Schrödinger equation discrete inverse scattering problem in polar coordinates with a nonlocal potential (a new result).

This paper is organized as follows. In section 2 we quickly review the equations of Newton's GLM machinery, which were shown to apply to the 2D case in [6]. We then use an inverse Radon transform to write these equations in the $x y$ domain. In section 3 we define a discrete 2D Laplacian operator and show that a linear system of equations with generalized Toeplitz-plus-Hankel structure produces a three-term recurrence that we recognize as a 2D discrete Schrödinger equation with nonlocal potential. We then develop discrete versions of the GLM integral equations. In section 4 we develop fast algorithms for solving these equations that we recognize as both discrete layer stripping algorithms and as fast split signal processing algorithms. Sections 3 and 4 modify the results of [17], and they can also be interpreted as a discrete implementation of the results of [8]. Section 5 concludes the paper by summarizing its results and their significance.

\section{Continuous two-dimensional Schrödinger inverse scattering equations}

\subsection{Review of basic equations}

We quickly review pertinent portions of Newton's GLM machinery for comparison with the discrete problem solved in section 3. It should be noted that the results of this paper are not limited to discretization of the equations of this section, but do demonstrate the results of such discretization. We follow [1-3]; [19,20] are good treatments.

The wavefield $\hat{u}(x, k)$ satisfies the Schrödinger equation

$$
\left(\Delta_{x}+k^{2}-V(x)\right) \hat{u}(x, k)=0
$$

where $x=\left(|x| \cos \theta_{x},|x| \sin \theta_{x}\right) \in R^{2}$ and the scattering potential $V(x)$ is real, smooth, and has compact support. It is also assumed that $V(x)$ does not induce bound states; a sufficient condition for this is for $V(x)$ to be non-negative. Two different sets of boundary conditions are specified, resulting in two different solutions.

The scattering solution $\psi\left(x, k, \theta_{i}\right)$ has boundary condition [6]

$$
\begin{aligned}
\psi\left(x, k, \theta_{i}\right)= & \mathrm{e}^{-\mathrm{i} k|x| \cos \left(\theta_{i}-\theta_{x}\right)}+A\left(k, \theta_{x}, \theta_{i}\right) \frac{\mathrm{e}^{-\mathrm{i} k|x|}}{\sqrt{|x|}}+\mathrm{O}\left(|x|^{-1}\right) \quad|x| \rightarrow \infty \\
= & \mathrm{e}^{-\mathrm{i} k|x| \cos \left(\theta_{i}-\theta_{x}\right)}-\int_{0}^{2 \pi} \mathrm{i} k A\left(k, \theta_{s}, \theta_{i}\right) \mathrm{e}^{-\mathrm{i} k|x| \cos \left(\theta_{s}-\theta_{x}\right)} \mathrm{d} \theta_{s}+\mathrm{O}\left(|x|^{-1}\right) \\
& |x| \rightarrow \infty
\end{aligned}
$$

where the scattering amplitude $A\left(k, \theta_{s}, \theta_{i}\right)$ is defined as

$$
A\left(k, \theta_{y}, \theta_{i}\right)=-\sqrt{8 \pi} \int \mathrm{e}^{\mathrm{i} k|y| \cos \left(\theta_{i}-\theta_{y}\right)} V(y) \psi\left(y, k, \theta_{i}\right) \mathrm{d} y
$$

and (2.2a) follows from (2.2b) by changing variables from $\theta_{s}$ to $s=|x| \cos \left(\theta_{s}-\theta_{x}\right)$. In the time domain this corresponds to an incident plane wave in the direction $\theta_{i}$ being used to probe the potential, and being scattered in all directions $\theta_{s}$. The scattering amplitude specifies the far-field behaviour of the wavefield, and constitutes the scattering data.

The regular solution $\phi\left(x, k, \theta_{i}\right)$ is defined as being the solution to (2.1) that is an entire analytic function of $k$ and is of exponential type $|x|$. It should be noted that, subject to mild assumptions [3], this solution generically exists and $\phi\left(x, k, \theta_{i}\right)-\mathrm{e}^{-\mathrm{i} k|x| \cos \left(\theta_{i}-\theta_{x}\right)}$ 
is square integrable in $k$. Using the Paley-Wiener theorem, its inverse Fourier transform $\check{\phi}\left(x, t, \theta_{i}\right)=\mathcal{F}^{-1}\left\{\phi\left(x, k, \theta_{i}\right)\right\}$ has support in $t$ on the interval $[-|x|,|x|]$. Thus, it has the Povsner-Levitan representation

$$
\phi\left(x, k, \theta_{i}\right)=\mathrm{e}^{-\mathrm{i} k|x| \cos \left(\theta_{i}-\theta_{x}\right)}-\int_{-|x|}^{|x|} m\left(x, t, \theta_{i}\right) \mathrm{e}^{-\mathrm{i} k t} \mathrm{~d} t
$$

where $m\left(x, t, \theta_{i}\right)$ is the nonimpulsive part of $\breve{\phi}\left(x, t, \theta_{i}\right)$.

The regular and scattering solutions are related by the Jost operator $J(k)$ squareintegrable on the unit circle with kernel $J\left(k, \theta_{1}, \theta_{2}\right)$ :

$$
\begin{aligned}
& \phi\left(x, k, \theta_{i}\right)=\int_{0}^{2 \pi} \psi\left(x, k, \theta_{s}\right) J\left(k, \theta_{s}, \theta_{i}\right) \mathrm{d} \theta_{s} \\
& \psi\left(x, k, \theta_{i}\right)=\int_{0}^{2 \pi} \phi\left(x, k, \theta_{s}\right) J^{-1}\left(k, \theta_{s}, \theta_{i}\right) \mathrm{d} \theta_{s} .
\end{aligned}
$$

Both $J(k)$ and $J^{-1}(k)$ are analytic in the lower half-plane (causality in the time domain).

We define the scattering operator with kernel

$$
\begin{aligned}
& S\left(k, \theta_{1}, \theta_{2}\right)=\delta\left(\theta_{1}-\theta_{2}\right)-\frac{\mathrm{i}}{\sqrt{2 \pi}} A\left(k, \theta_{1}, \theta_{2}\right) \\
& \psi\left(x, k, \theta_{1}\right)=\int_{0}^{2 \pi} S\left(k, \theta_{1}, \theta_{2}\right) \psi\left(x,-k, \theta_{2}+\pi\right) \mathrm{d} \theta_{2}
\end{aligned}
$$

and $Q$ as the operator such that $Q A\left(k, \theta_{1}, \theta_{2}\right)=A\left(k, \theta_{1}+\pi, \theta_{2}\right)$. We write (2.5) and (2.6) in operator notation as [1] (note the order indicates which $\theta$ is integrated)

$$
\phi=\psi J \quad \psi=\phi J^{-1} \quad S=1-\frac{\mathrm{i}}{\sqrt{2 \pi}} A \quad \psi(k)=S \psi(-k) Q .
$$

\subsection{Review of Gel'fand-Levitan and Marchenko integral equations}

The Marchenko procedure for solving the inverse scattering problem is as follows. Given the scattering amplitude $A\left(k, \theta_{s}, \theta_{i}\right)$, compute

$$
G\left(t, \theta_{s}, \theta_{i}, x\right)=\frac{1}{2 \pi} \int_{-\infty}^{\infty} \mathrm{i} k \mathrm{e}^{\mathrm{i} k\left(t-|x|\left(\cos \left(\theta_{s}-\theta_{x}\right)-\cos \left(\theta_{i}-\theta_{x}\right)\right)\right)} A\left(k, \theta_{s}, \theta_{i}\right) \mathrm{d} k
$$

and then solve the generalized Marchenko equation $[1,6]$

$v\left(x, t, \theta_{i}\right)=\int_{0}^{2 \pi} G\left(t, \theta_{s}, \theta_{i}, x\right) \mathrm{d} \theta_{s}+\int_{0}^{\infty} \int_{0}^{2 \pi} G\left(t-\tau, \theta^{\prime}, \theta_{i}, x\right) v\left(x,-\tau,-\theta^{\prime}\right) \mathrm{d} \theta^{\prime} \mathrm{d} \tau$

for the delayed scattered field

$$
\begin{aligned}
& v\left(x, t, \theta_{i}\right)=u\left(x, t-|x| \cos \left(\theta_{i}-\theta_{x}\right), \theta_{i}\right) \\
& u\left(x, t, \theta_{i}\right)=\psi\left(x, t, \theta_{i}\right)-\delta\left(t-|x| \cos \left(\theta_{i}-\theta_{x}\right)\right) .
\end{aligned}
$$

The potential $V(x)$ is then recovered from the $v\left(x, t, \theta_{i}\right)$ using the miracle equation $[1,6]$

$$
\begin{aligned}
& V(x)=2\left(\cos \theta_{i}, \sin \theta_{i}\right) \cdot \nabla v\left(x, t=0, \theta_{i}\right) \\
& =2\left(\cos \theta_{i}, \sin \theta_{i}\right) \cdot \nabla u\left(x, t=|x| \cos \left(\theta_{i}-\theta_{x}\right), \theta_{i}\right) .
\end{aligned}
$$

Note that the right-hand side of (2.11) must be independent of the direction of incidence $\theta_{i}$. This is the 'miracle', and it imposes a constraint on $\psi\left(x, k, \theta_{i}\right)$ and hence on $A\left(k, \theta_{s}, \theta_{i}\right)$, since this has five degrees of freedom and the potential $V(x)$ generating it has only three 
degrees of freedom. There is no known simple test to see if a $A\left(k, \theta_{s}, \theta_{i}\right)$ results in a 'miracle', although one known result is [21].

The Gel'fand-Levitan procedure for solving the inverse scattering problem is as follows. Compute $J^{-1}\left(k, \theta_{1}, \theta_{2}\right)$ from scattering data $A\left(k, \theta_{s}, \theta_{i}\right)$ by solving the integral equation

$L\left(t, \theta_{s}, \theta_{i}\right)=G\left(t, \theta_{s}+\pi, \theta_{i}, 0\right)+\int_{0}^{\infty} \int_{0}^{2 \pi} L\left(\tau, \theta_{s}+\pi, \theta^{\prime}\right) G\left(t+\tau, \theta^{\prime}, \theta_{i}, 0\right) \mathrm{d} \theta^{\prime} \mathrm{d} \tau$

where $G\left(t, \theta_{s}, \theta_{i}, x\right)$ is defined in (2.8). Although (2.12) looks like (2.9), note that (2.12) need only be solved for $x=0$, while (2.9) must be solved for all $x$. We then have

$$
J^{-1}\left(k, \theta_{1}, \theta_{2}\right)=1+\int_{0}^{\infty} L\left(t, \theta_{1}, \theta_{2}\right) \mathrm{e}^{-\mathrm{i} k t} \mathrm{~d} t .
$$

Now define

$$
M\left(t, \theta_{1}, \theta_{2}\right)=\mathcal{F}^{-1}\left\{\left[\left(J^{H} J\right)^{-1}-\delta\left(\theta_{1}-\theta_{2}\right)\right]\right\}
$$

as the inverse Fourier transform of the perturbation of the spectral function away from its free-space representation. Then the nonimpulsive part $m\left(x, t, \theta_{i}\right)$ of the regular solution $\phi\left(x, t, \theta_{i}\right)$ may be obtained by solving the Gel'fand-Levitan integral equation [3]

$$
\begin{aligned}
m\left(x, t, \theta_{i}\right)= & \int_{0}^{2 \pi} M\left(t+|x| \cos \left(\theta_{s}-\theta_{x}\right), \theta_{s}, \theta_{i}\right) \mathrm{d} \theta_{s} \\
& -\int_{0}^{2 \pi} \int_{-|x|}^{|x|} m\left(x, \tau, \theta_{s}\right) M\left(t+\tau, \theta_{s}, \theta_{i}\right) \mathrm{d} \tau \mathrm{d} \theta_{s}
\end{aligned}
$$

and the scattering potential $V(x)$ may be recovered from $m\left(x, t, \theta_{i}\right)$ with

$$
V(x)=2\left(\cos \theta_{i}, \sin \theta_{i}\right) \cdot \nabla m\left(x, t=|x| \cos \left(\theta_{i}-\theta_{x}\right), \theta_{i}\right) .
$$

This concludes our review of multidimensional inverse scattering theory.

\subsection{Transformation to xy domain: Gel'fand-Levitan equation}

The aim of this section is to use the inverse Radon transform to map the above equations from the $\left(x, t, \theta_{i}\right)$ domain to the $(x, y)$ domain. To do this will require some processing of the above equations.

First, note the regular solution $\phi\left(x, k, \theta_{i}\right)$ and its nonimpulsive part $m\left(x, t, \theta_{i}\right)$ defined in (2.4) have the properties $\phi\left(x, k, \theta_{i}\right)=\phi\left(x,-k, \theta_{i}+\pi\right)$ and $m\left(x, t, \theta_{i}\right)=m\left(x,-t, \theta_{i}+\pi\right)$. Hence, we may regard $\phi\left(x, k, \theta_{i}\right)$ as the 2D Fourier transform of some function $\delta(x-y)-$ $h(x, y)$ since $\mathcal{F}_{y \rightarrow\left(k, \theta_{i}\right)}\{\delta(x-y)\}=\mathrm{e}^{-\mathrm{i} k|x| \cos \left(\theta_{i}-\theta_{x}\right)}$. Alternatively, we may regard $h(x, y)$ as the inverse Radon transform of $m\left(x, t, \theta_{i}\right)$. The Radon transform is defined as

$$
\begin{aligned}
& \mathcal{R}\{f(y)\}=\hat{f}(t, \theta)=\int_{R^{2}} f(y) \delta\left(t-|y| \cos \left(\theta-\theta_{y}\right)\right) \mathrm{d} y \\
& f(y)=\frac{1}{4 \pi} \int_{-\infty}^{\infty} \int_{0}^{2 \pi} \mathcal{H} \frac{\mathrm{d}}{\mathrm{d} t} \hat{f}(t, \theta) \delta\left(t-|y| \cos \left(\theta-\theta_{y}\right)\right) \mathrm{d} \theta \mathrm{d} t
\end{aligned}
$$

where $\mathcal{H}$ is the Hilbert transform (convolution with $1 /(\pi t)$ ). So we define

$-h(x, y)=\mathcal{F}_{\left(k, \theta_{i}\right) \rightarrow y}^{-1}\left\{\phi\left(x, k, \theta_{i}\right)-\mathrm{e}^{-\mathrm{i} k|x| \cos \left(\theta_{i}-\theta_{x}\right)}\right\}=\mathcal{R}_{\left(t, \theta_{i}\right) \rightarrow y}^{-1}\left\{m\left(x, t, \theta_{i}\right)\right\}$.

Since $m\left(x, t, e_{i}\right)$ has support in $t$ on the interval $[-|x|,|x|], h(x, y)$ has support inside the sphere $|y| \leqslant|x|$, as long as $m\left(x, t, e_{i}\right)$ satisfies mild conditions (the triangularity of $h(x, y)$ has been shown in [3]). 
Taking the partial inverse Radon transform [3, p 601] of the generalized Gel'fandLevitan equation (2.15) results in $[1,3]$

$k(x, y)=h(x, y)+\int_{|z| \leqslant|x|} h(x, z) k(z, y) \mathrm{d} z \quad|y| \leqslant|x|$
$k(x, y)=\frac{1}{(2 \pi)^{2}} \int_{0}^{\infty} \int_{0}^{2 \pi} \int_{0}^{2 \pi} M\left(k, \theta_{1}, \theta_{2}\right) \mathrm{e}^{-\mathrm{i} k\left(|x| \cos \left(\theta_{1}-\theta_{x}\right)-|y| \cos \left(\theta_{2}-\theta_{y}\right)\right)} k^{2} \mathrm{~d} \theta_{1} \mathrm{~d} \theta_{2} \mathrm{~d} k$

where $M\left(k, \theta_{1}, \theta_{2}\right)$ was defined in (2.14). Note that in the $x y$ domain the Gel'fand-Levitan equation is a $2 \mathrm{D}$ Wiener-Hopf equation.

Also, from (2.20) we see that

$\delta(x-y)+k(x, y)=\mathcal{F}_{\left(k_{1}, \theta_{1}\right) \rightarrow x}^{-1} \mathcal{F}_{\left(k_{2}, \theta_{2}\right) \rightarrow y}^{-1}\left\{\left(J^{H} J\right)^{-1}\left(k_{1}, \theta_{1}, \theta_{2}\right) \delta\left(\left|k_{1}\right|-\left|k_{2}\right|\right)\right\}$.

This shows that $\delta(x-y)+k(x, y)$ is a positive definite function, and that the 2D Fourier transform of $k(x, y)$ is zero except for the on-shell values for which $\left|k_{1}\right|=\left|k_{2}\right|$. This structure in the wavenumber domain implies that the function $k(x, y)$ has structure [3]

$$
\left(\Delta_{x}-\Delta_{y}\right) k(x, y)=0
$$

where $\Delta_{x}$ is the Laplacian with respect to $x$.

Finally, taking the inverse Fourier transform of the Schrödinger equation (2.1) with $\hat{u}(x, k)=\phi\left(x, k, \theta_{i}\right)$ results in [3]

$$
\left(\Delta_{x}-\Delta_{y}\right) h(x, y)=V(x) h(x, y) \quad V(x)=-\frac{2}{|x|} \frac{\mathrm{d}}{\mathrm{d}|x|}|x| h(x, y=x) .
$$

This means that the inverse scattering problem can be reformulated as follows. Given $M\left(t, \theta_{1}, \theta_{2}\right)$ as defined in (2.14), compute its double inverse Radon transform $k(x, y)$ using (2.20) and solve the integral equation (2.19). $V(x)$ is recovered from the solution $h(x, y)$ using (2.23) instead of (2.16).

Alternatively, we can derive the Gel'fand-Levitan equation directly in the $x y$ domain (2.19) as in [8] and [16]. Applying the operator $\left(\Delta_{x}-\Delta_{y}\right)$ to (2.19) and using (2.22), we obtain directly (2.23). Since $k(x, y)$ is positive definite, (2.19) has a unique solution [22], and this must be the nonimpulsive part $h(x, y)$ of the regular solution satisfying the Schrödinger equation in the $x y$ domain. Conversely, we may find this solution by solving the integral equation.

We can propagate (2.23) as a continuous-domain layer stripping algorithm, computing $V(x)$ as we go. We initialize $h(0,0)=0$ and $h(\Delta, y)$ known from direct solution of (2.19) (this is a small problem compared with that for larger $|x|)$. We then propagate (2.23) in increasing $|x|$, computing $V(x)$ at each $|x|$ using (2.23) and using that $V(x)$ to propagate (2.23) to $|x|+\Delta$ for some small $\Delta$. We must obtain $h(x, x)$ from $h(x, y)$ using (2.19). This is the continuous version of the discrete split Levinson algorithm of section 4.1.

\subsection{Transformation to $x y$ domain}

Most of section 2.3 appeared somewhere in $[1-3,7-10,16]$. The material to follow is entirely new.

We cannot repeat this approach on the scattering solution $\psi(x, k, \theta)$ since $\psi(x, k, \theta) \neq$ $\psi(x,-k, \theta+\pi)$. Instead we consider (scattered field $u\left(x, t, \theta_{i}\right)$ is defined in (2.10))

$$
\begin{aligned}
& \tilde{\psi}(x, k, \theta)=\psi(x, k, \theta)+\psi(x,-k, \theta+\pi) \\
& \tilde{u}\left(x, t, \theta_{i}\right)=u\left(x, t, \theta_{i}\right)+u\left(x,-t, \theta_{i}+\pi\right) .
\end{aligned}
$$


Since $\tilde{u}\left(x, t, \theta_{i}\right)=\tilde{u}\left(x,-t, \theta_{i}+\pi\right)$, we can define the inverse Radon transforms

$$
\begin{aligned}
& u(x, y)=\mathcal{R}_{\left(t, \theta_{i}\right) \rightarrow y}^{-1}\left\{\tilde{u}\left(x, t, \theta_{i}\right)\right\} \\
& R(x, y)=\mathcal{R}_{\left(t_{1}, \theta_{1}\right) \rightarrow x}^{-1} \mathcal{R}_{\left(t_{2}, \theta_{2}\right) \rightarrow y}^{-1}\left\{\delta\left(t_{1}-t_{2}\right) \tilde{G}\left(t_{1}, \theta_{1}, \theta_{2}, x\right)\right\}
\end{aligned}
$$

where $\tilde{G}\left(t_{1}, \theta_{1}, \theta_{2}, x\right)$ is defined as in (2.24) and $R(x, y)$ satisfies (2.22).

$u(x, y)$ can be related to the Gel'fand-Levitan equation solution as follows. Changing $k$ to $-k$ and $\theta$ to $\theta+\pi$ throughout (2.5) and adding to (2.5) gives (recall $\phi(x, k, \theta)=$ $\phi(x,-k, \theta+\pi))$

$\tilde{\psi}\left(x, k_{1}, \theta_{1}\right)=\int_{0}^{2 \pi} \int_{0}^{\infty} \phi\left(x, k_{2}, \theta_{2}\right) \tilde{J}^{-1}\left(k_{2}, \theta_{2}, \theta_{1}\right) \delta\left(\left|k_{1}\right|-\left|k_{2}\right|\right) \mathrm{d} k_{2} \mathrm{~d} \theta_{2}$

where $\tilde{J}^{-1}$ is defined as in (2.24). Taking inverse Fourier transforms and using Parseval's theorem gives, as in (2.21) and interpreting $\int \delta(x-y) \delta(x-z) \mathrm{d} x=\delta(y-z)$,

$$
\tilde{\psi}(x, y)=\int \tilde{\phi}(x, z) J^{-1}(z, y) \mathrm{d} z
$$

where $\tilde{\psi}(x, y)$ and $\tilde{J}^{-1}(x, y)$ are the obvious inverse Fourier transforms and $\tilde{J}^{-1}(x, y)$, like $R(x, y)$ and $k(x, y)$, satisfies (2.22). By linearity, $\tilde{\psi}(x, y)$ satisfies (2.23) since $\phi(x, y)$ does. Note that both $\phi(x, y)$ and $\tilde{\psi}(x, y)$ satisfy the first equation of (2.23), while their nonimpulsive parts satisfy both equations of (2.23).

The boundary condition for $\tilde{\psi}(x, y)$ is found from $(2.2 b)$ and $(2.8)$ as

$$
\begin{aligned}
& \tilde{\psi}(x, y)=2 \delta(x-y)+R(x, y)+\mathrm{O}\left(|x|^{-1}\right) \quad|x| \rightarrow \infty \\
& R(x, y)=\mathcal{F}_{\left(k_{1}, \theta_{1}\right) \rightarrow x}^{-1} \mathcal{F}_{\left(k_{2}, \theta_{2}\right) \rightarrow y}^{-1}\left\{\tilde{A}\left(k_{1}, \theta_{1}, \theta_{2}\right) \delta\left(\left|k_{1}\right|-\left|k_{2}\right|\right)\right\} \\
& \tilde{A}\left(k, \theta_{s}, \theta_{i}\right)=A\left(k, \theta_{s}, \theta_{i}\right)+A\left(-k, \theta_{s}+\pi, \theta_{i}+\pi\right)=A\left(k, \theta_{s}, \theta_{i}\right)+A\left(-k, \theta_{i}, \theta_{s}\right)
\end{aligned}
$$

using reciprocity [3] (the impulse is also doubled). Setting $x=0$ in (2.27) also gives $\tilde{\psi}(0, y)=\tilde{J}^{-1}(0, y)$. This provides another reformulation of the inverse scattering problem, in which the problem is solved in increasing $|x|$ rather than decreasing $|x|$ (see below).

We now show that $u(x, y)$ satisfies an equation like (2.23). To see this, note

$$
\left(\Delta_{x}+k^{2}-V(x)\right) \tilde{\psi}(x, k, \theta)=0
$$

from (2.24), since both $\psi(x, k, \theta)$ and $\psi(x,-k, \theta+\pi)$ satisfy the Schrödinger equation (the latter is denoted $\psi^{-}$in [1]). Since $\tilde{\psi}(x, k, \theta)=\tilde{\psi}(x,-k, \theta+\pi)$, we can take an inverse Fourier transform taking $(k, \theta)$ into $y$. The result is

$$
\left(\Delta_{x}-\Delta_{y}\right) u(x, y)=V(x) u(x, y) .
$$

Letting $|x| \rightarrow \infty$ in $(2.28 a)$ shows that $u(x, y) \simeq R(x, y)$, although it should be noted that we cannot be sure $u(x, y) \simeq 0$ since $R(x, y)$ may have a singularity in $|y|$.

We now show that we can set $u(x, y)=0$ for $|y|<|x|$. Note that:

(1) Equation (2.30) has a characteristic $|y|=|x|$, and $\{u(x, y),|y|>|x|\}$ propagates in $|x|$ entirely independently of $\{u(x, y),|y|<|x|\}$.

(2) The miracle equation (2.11) still holds when $u\left(x, t, \theta_{i}\right)$ is replaced with $\tilde{u}\left(x, t, \theta_{i}\right)$, since both $\nabla u\left(x, t, \theta_{i}\right)$ and $\nabla u\left(x,-t, \theta_{i}+\pi\right)$ jump from zero at $t=|x| \cos \left(\theta_{i}-\theta_{x}\right)$, so their sum $\nabla \tilde{u}\left(x, t, \theta_{i}\right)$ has this value.

(3) Modifying the argument in appendix B of [8] gives the miracle equation (2.11) in the $x y$ domain (note the sign change from (2.23)). We have

$$
V(x)=2\left(\cos \theta_{i}, \sin \theta_{i}\right) \cdot \nabla \tilde{u}\left(x, t=|x| \cos \left(\theta_{i}-\theta_{x}\right), \theta_{i}\right)=\frac{2}{|x|} \frac{\mathrm{d}}{\mathrm{d}|x|}|x| u(x, y=x) .
$$


Note that neither (2.31) nor propagation of $\{u(x, y),|y|>|x|\}$ in (2.30) requires $\{u(x, y),|y|<|x|\}$. Hence, for the inverse scattering problem, we may set the latter to zero.

Note that for the 3D problem the nonlocal derivative-Hilbert transform operator in the inverse Radon transform $(2.17 b)$ becomes a local second derivative. Then, setting $u(x, y)=0$ for $|y|<|x|$ is equivalent to setting $\tilde{u}\left(x, t, \theta_{i}\right)=0$ for $|t|<|x|$, so that triangularity is induced in the time domain as well as the $x y$-domain. In the $2 \mathrm{D}$ problem the nonlocal derivative-Hilbert transform precludes this; $\tilde{u}\left(x, t, \theta_{i}\right)$ for $|t|<|x|$ is whatever values are required to make $u(x, y)=0$ for $|y|<|x|$. These values do not affect $\{u(x, y),|y|>|x|\}$ since these are determined by $\left\{\tilde{u}\left(x, t, \theta_{i}\right),|t|>|x|\right\}$.

\subsection{Continuous layer stripping algorithms}

We can propagate (2.30) as a continuous-domain layer stripping algorithm, computing $V(x)$ as we go, initialized in any of three different ways.

(1) At $x=0$ using $u(0, y)=\tilde{J}^{-1}(0, y)$ if this factor is known. We then propagate (2.30) in increasing $|x|$, computing $V(x)$ at each $|x|$ using (2.31) and using that $V(x)$ to propagate (2.30) to $|x|+\Delta$ for some small $\Delta$.

(2) At $|x| \rightarrow \infty$ using $u(x, y) \simeq R(x, y)$ if the scattering amplitude is known. We then propagate (2.30) in decreasing $|x|$, computing $V(x)$ at each $|x|$ using (2.31) and using $V(x)$ to propagate (2.30) to $|x|-\Delta$. This is the continuous version of the discrete split lattice algorithm of section 4.3 .

(3) At finite $|x|$ outside the support of $V(x)$ if the near-field scattered field is known. We then proceed in decreasing $|x|$ as in (2). This is also the continuous version of the discrete split lattice algorithm.

Of course this is inefficient; the backscattered field due to probing in a single direction $\theta_{i}$ is sufficient to reconstruct $V(x)[13-15,23]$. However, here we are deriving results for the Newton GLM machinery.

One final operation is required before passing to the discrete problem. The Laplacian operator in polar coordinates $(r, \theta)$ is

$\Delta f(r, \theta)=\frac{1}{r} \frac{\partial}{\partial r} r \frac{\partial f}{\partial r}+\frac{1}{r^{2}} \frac{\partial^{2} f}{\partial \theta^{2}}=\frac{1}{\sqrt{r}} \frac{\partial^{2}}{\partial r^{2}}(\sqrt{r} f)+\frac{1}{4 r^{2}} f+\frac{1}{r^{2}} \frac{\partial^{2} f}{\partial \theta^{2}}$.

The $1 /\left(4 r^{2}\right)$ term does not appear in the Laplacian operator in an odd number of dimensions; it shows the Green function in an even number of dimensions is only asymptotically an impulse. Using (2.32), we rewrite (2.23) as

$$
\begin{gathered}
\left(\frac{\partial^{2}}{\partial|x|^{2}}+\frac{1}{4|x|^{2}}+\frac{1}{|x|^{2}} \frac{\partial^{2}}{\partial \theta_{x}^{2}}-\frac{\partial^{2}}{\partial|y|^{2}}-\frac{1}{4|y|^{2}}-\frac{1}{|y|^{2}} \frac{\partial^{2}}{\partial \theta_{y}^{2}}\right) \sqrt{|x||y|} h(x, y) \\
=V(x) \sqrt{|x||y|} h(x, y) \\
V(x)=-\left(\frac{\partial}{\partial|x|}+\frac{\partial}{\partial|y|}\right) h(x, y=x) .
\end{gathered}
$$

\subsection{Summary}

The inverse scattering problem can be formulated as follows. The basic equation to be solved is (2.1), which transforms into (2.33) as noted above. Given $M\left(t, \theta_{1}, \theta_{2}\right)$ as defined in (2.14), compute its double inverse Radon transform $k(x, y)$ using (2.20) and solve the Gel'fand-Levitan integral equation (2.19). $V(x)$ is recovered from the solution $h(x, y)$ using 
(2.23). Or, given scattering amplitude $A\left(k, \theta_{s}, \theta_{i}\right)$, find $G\left(t, \theta_{1}, \theta_{2}, x\right)$ using (2.8), compute the double inverse Radon transform $R(x, y)$ using (2.28) and run any of the continuous layer stripping algorithms of section 2.5.

We note at this point that the 2D Wiener-Hopf equation (2.19) leads to stochastic interpretations of all of these equations [16]. $h(x, y)$ is the optimal filter for computing the linear least-squares estimate of a zero-mean random field with covariance $k(x, y)$ at the boundary of a circle of noisy measurements. Also, the fact that $k(x, y)$ (along with $\tilde{J}^{-1}(x, y)$ and $\left.R(x, y)\right)$ has the generalized Toeplitz-plus-Hankel structure (2.22) (see [8]) makes possible all of the fast layer stripping algorithms. We show this in the discrete case explicitly.

\section{Discrete two-dimensional Schrödinger inverse scattering equations}

\subsection{Formulation of two-dimensional discrete inverse scattering problem}

In this section the discrete counterpart to section 2 is considered. We replace the continuous 2D Laplacian operator (2.32) with its discrete counterpart

$$
\begin{aligned}
\Delta f(i, m)= & \frac{f^{\prime}(i+1, m)+f^{\prime}(i-1, m)-2 f^{\prime}(i, m)}{\sqrt{i}} \\
& +\frac{f(i, m)}{4 i^{2}}+\frac{f(i, m+1)+f(i, m-1)-2 f(i, m)}{i^{2}}
\end{aligned}
$$

where $i$ denotes radial and $m$ denotes angular indices and $f^{\prime}(i, m)=\sqrt{i} f(i, m)$. We can regard (3.1) as a discretization $r=i \Delta_{r}, \theta=m \Delta_{\theta}$ of (2.32), or as an explicitly discrete operator. We omit factors of $\Delta_{r}$ and $\Delta_{\theta}$ throughout. Using (3.1), rewrite (2.33) as

$$
\begin{aligned}
h(i+1, j, m, n) & +h(i-1, j, m, n)-h(i, j+1, m, n)-h(i, j-1, m, n) \\
& +\frac{h(i-1, j, m+1, n)+h(i-1, j, m-1, n)-2 h(i-1, j, m, n)}{i^{2}} \\
& -\frac{h(i-1, j, m, n+1)+h(i-1, j, m, n-1)-2 h(i-1, j, m, n)}{j^{2}} \\
& +\frac{h(i, j, m, n)}{4 i^{2}}-\frac{h(i, j, m, n)}{4 j^{2}} \\
= & \sum_{k=0}^{M-1} V(i, m, k) h(i-1, j, k, n)
\end{aligned}
$$

where $h(i, j, m, n)$ can be viewed as a discretization of $\sqrt{|x||y|} h(x, y)$ in which $|x|=i \Delta_{r}$, $|y|=j \Delta_{r}, \theta_{x}=m \Delta_{\theta}$, and $\theta_{y}=n \Delta_{\theta}$ and $\Delta_{\theta}=2 \pi / M$. Note that the angular indices $m$ and $n$ are periodic with period $M$. Also note that the potential $V(x)$ in $(2.33)$ has been replaced with a discrete nonlocal potential $V(i, m, n)$ in (3.2); this will correspond to (2.33) if $V(i, m, n)=V(i, m) \delta(m-n)$ where $\delta(i-j)$ is now a discrete impulse. However, we will show that in fact a nonlocal potential will almost always be the result of solving the inverse scattering problem, which is why we incorporate it into (3.2).

The discrete 2D inverse scattering problem is defined as follows. Given $k(i, j, m, n)$ or $R(i, j, m, n)$, compute the scattering potential $V(i, m)$. This can be viewed either as a discretization of the continuous problem, or as an explicitly discrete problem.

We define the regular solution to the discrete $2 \mathrm{D}$ inverse scattering problem as the solution satisfying $\phi(0, j, m, n)=\delta(j)$ (note angular indices $m$ and $n$ are irrelevant when the radii $i=j=0)$. $\phi(i, j, m, n)$ has support on $i \geqslant j \geqslant 0$. The scattering solution 
$\psi(i, j, m, n)$ can be defined using $\psi(0, j, m, n)=J^{-1}(0, j, m, n)$; it satisfies asymptotic conditions analogous to (2.2) and (2.32). The smooth parts (so to speak) $h(i, j, m, n)$ and $u(i, j, m, n)$ of $\phi(i, j, m, n)$ and $\psi(i, j, m, n)$, respectively, are defined as before with a discrete impulse. The condition (2.22) satisfied by $k(x, y), R(x, y)$, and $\tilde{J}^{-1}(x, y)$ becomes the discrete condition (3.2) with the right-hand side set equal to zero.

\subsection{Discrete two-dimensional Gel'fand-Levitan procedure}

The discrete counterpart to the Gel'fand-Levitan equation (2.19) is

$$
k(i, j, m, n)=h(i, j, m, n)+\sum_{k=0}^{i-1} \sum_{l=0}^{M-1} h(i, k, m, l) k(k, j, l, n) .
$$

Recall that the radial weighting factor $\sqrt{|x||y|}$ has been incorporated into $h(i, j, m, n)$ and $k(i, j, m, n)$, and this accounts for the absence of radial weight $k$ in (3.3). However, we impose a subtle condition on the sum over $k$ : it only includes indices $k$ that have the same parity (even or odd) as $i-1$. For example, the sum may include values $k=1,3,5,7,9=i-1$ or $k=0,2,4,6,8=i-1$, skipping every other value. The sum over index $l$ does not skip any values. This peculiar arrangement does not make any difference in the continuous limit $\Delta \rightarrow 0$, but it is vital in the discrete case; this is the first of many points on which simple discretization of the continuous equations will not work. The skipping-index arrangement replaces the use of interpolated systems in [17].

We now modify an argument we used in [17]. Apply the discrete operator defined by the left-hand side of (3.2) (the discrete counterpart to $\left(\Delta_{x}-\Delta_{y}\right)$ ) to (3.3). After some algebra and using the discrete counterpart to (2.22), we obtain precisely (3.2)! We also obtain

$$
V(i, m, n)=h(i+1, i, m, n)-h(i, i-1, m, n)
$$

which is clearly a discrete counterpart to the second equation of (2.33)!

We now present some comments on the algebra involved in deriving (3.2) and (3.4) from (3.3).

(1) The basic idea is to apply the discrete $\left(\Delta_{x}-\Delta_{y}\right)$ to (3.3) and obtain an equation similar to (3.3), except that $h(i, j, m, n)$ is replaced by $\left(\Delta_{x}-\Delta_{y}\right) h(i, j, m, n)$ and $k(i, j, m, n)$ is replaced by a multiple of $h(i-1, j, m, n)$; the multiple defines $V(i, m, n)$. Since $\delta(i-j)+k(i, j, m, n)$ is positive definite, (3.3) has a unique solution, and the new solution must be the old solution multiplied by $V(i, m, k)$ and summed over index $k$.

(2) Most of the algebra was given in [17] and will not be repeated here. The following changes are required:

(a) the additional term is handled by multiplying (3.3) by

$$
\frac{1}{4 i^{2}}-\frac{1}{4 j^{2}}=\left(\frac{1}{4 i^{2}}-\frac{1}{4 k^{2}}\right)+\left(\frac{1}{4 k^{2}}-\frac{1}{4 j^{2}}\right)
$$

and adding the result to the argument in [17];

(b) since the radial indices $i$ and $j$ are not changing, the radial factor $1 / i^{2}$ multiplying the angular part is also handled using (3.5);

(c) the half-integer indices $i$ and $j$ become integers as above.

(3) Note the angular parts of the Laplacians are both defined at $i-1$ instead of $i$, and the potential multiplies $h(i-1, j, m, n)$, not $h(i, j, m, n)$. These seemingly innocuous changes, negligible in the continuous limit, are necessary in the discrete case.

(4) The above result is only exact if the angular and radial parts of the difference of discrete Laplacians $\left(\Delta_{x}-\Delta_{y}\right) k(x, y)$ are separately zero [17]. Since a rotation in the 
spatial domain produces a rotation in the Fourier domain, this happens if the spectral function $M\left(t, \theta_{1}, \theta_{2}\right)$ defined in (2.14) has Toeplitz-plus-Hankel structure in $\theta_{1}, \theta_{2}$, i.e. $M\left(t, \theta_{1}, \theta_{2}\right)=M_{1}\left(t, \theta_{1}-\theta_{2}\right)+M_{2}\left(t, \theta_{1}+\theta_{2}\right)$ for some functions $M_{1}(\cdot), M_{2}(\cdot)$. Otherwise the derivation in [17] requires at one point approximating $h(i, j, m, n)$ with $h(i-1, j, m, n)$, which is valid in the limit $\Delta \rightarrow 0$.

The above argument shows that the solution to the discrete Gel'fand-Levitan equation (3.3) satisfies (3.2) with $h(0, j, m, n)=0$. This means $h(i, j, m, n)$ is the 'smooth' part of the regular solution (the regular solution minus a discrete impulse), since the regular solution is unique. The discrete potential can be recovered from $h(i, j, m, n)$ using (3.4). Thus, the entire continuous Gel'fand-Levitan procedure has an exact discrete counterpart, and the latter, unlike the former, can be directly implemented numerically. However, while the continuous results can easily be obtained from the discrete results by letting $\Delta \rightarrow 0$, the continuous equations will not work unless they are properly discretized (3.2), (3.3).

\subsection{Discrete two-dimensional Marchenko procedure}

A similar argument can be used to derive a discrete Marchenko procedure. We define the discrete Marchenko equation to be (compare with (3.3))

$$
R(i, j, m, n)=u(i, j, m, n)+\sum_{k=i+1}^{\infty} \sum_{l=0}^{M-1} u(i, k, m, l) R(k, j, l, n) .
$$

Again the radial weighting factor $\sqrt{|x||y|}$ has been incorporated into $u(i, j, m, n)$ and $R(i, j, m, n)$, and the sum over $k$ includes only indices $k$ having the same parity (even or odd) as $i+1$. Again applying the discrete counterpart of $\left(\Delta_{x}-\Delta_{y}\right)$ to (3.6) and modifying the argument used in [17] (the sum now starts at $k=i+1$ instead of ending at $k=i-1)$, we obtain

$$
\begin{aligned}
u(i+1, j, m, n) & +u(i-1, j, m, n)-u(i, j+1, m, n)-u(i, j-1, m, n) \\
+ & \frac{u(i+1, j, m+1, n)+u(i+1, j, m-1, n)-2 u(i+1, j, m, n)}{i^{2}} \\
& -\frac{u(i+1, j, m, n+1)+u(i+1, j, m, n-1)-2 u(i+1, j, m, n)}{j^{2}} \\
& +\frac{u(i, j, m, n)}{4 i^{2}}-\frac{u(i, j, m, n)}{4 j^{2}} \\
= & \sum_{k=0}^{M-1} V(i, m, k) u(i+1, j, k, n) \\
V(i, m, n)= & u(i-1, i, m, n)-u(i, i+1, m, n) .
\end{aligned}
$$

Comparing (3.7) with (3.2), note that all of the $h(i-1, j, m, n)$ have been replaced with $u(i+1, j, m, n)$, and that (3.8) is clearly a discrete counterpart to (2.30).

Comments on this approach are identical to those for the Gel'fand-Levitan equation. The only major difference is the infinite range of the summation in (3.6), which requires that we assume that the sum converges, so that we can define a system of equations of infinite order with a unique solution.

\section{Discrete layer stripping algorithms}

The 2D discrete inverse scattering problem can also be solved by using fast layer stripping algorithms. In the discrete case, we can show that these layer stripping algorithms also 
directly solve the Gel'fand-Levitan and Marchenko systems of equations in section 3. Indeed, the layer stripping algorithms can now be seen to be classical split fast signal processing algorithms for solving these structured systems of equations.

An early form of layer stripping algorithm is the dynamic deconvolution concept developed in the 1950s in the oil industry [18]. These algorithms have the reputation of being numerically unstable when applied to noisy data. We have shown that this is due to their being fed infeasible data, data that could not possibly have come from a scattering medium as modelled. If the noisy data are corrected to become feasible (although still noisy) the forms of layer stripping that correspond to classical signal processing algorithms such as the Levinson and Schur algorithms are numerically stable.

The layer stripping approach and the GLM integral equation approach have been shown to be related in the $1 \mathrm{D}$ case $[11,12]$ and the $3 \mathrm{D}$ case $[5,7,8,16]$. However, numerical implementation requires discrete algorithms, and as we have shown above determining the proper discrete algorithm is not trivial. Here we provide explicitly discrete layer stripping algorithms that solve the 2D discrete inverse scattering problem. We also show that these are fast split signal processing algorithms for the Gel'fand-Levitan and Marchenko matrix equations, so the two approaches are not only related but identical in the discrete case.

\subsection{Split Levinson-type algorithm}

To compute the potential $V(i, m, n)$, define the Schur variables

$$
\begin{aligned}
s(i, j, m, n)= & \delta(i-j) \delta(m-n)+k(i, j, m, n)-h(i, j, m, n) \\
& -\sum_{k=0}^{i-1} \sum_{l=0}^{M-1} h(i, k, m, l) k(k, j, l, n) .
\end{aligned}
$$

From (3.3) it is clear that $s(i, j, m, n)=0$ for $i>j$; compare this with $h(i, j, m, n)=0$ for $i \leqslant j$. Since $s(i, j, m, n)$ is a linear combination of $\delta(i-j) \delta(m-n)-h(i, j, m, n)$, $s(i, j, m, n)$ satisfies (3.2) for all $j$ :

$$
\begin{aligned}
s(i+1, j, m, n) & +s(i-1, j, m, n)-s(i, j+1, m, n)-s(i, j-1, m, n) \\
+ & \frac{s(i-1, j, m+1, n)+s(i-1, j, m-1, n)-2 s(i-1, j, m, n)}{i^{2}} \\
& -\frac{s(i-1, j, m, n+1)+s(i-1, j, m, n-1)-2 s(i-1, j, m, n)}{j^{2}} \\
& +\frac{s(i, j, m, n)}{4 i^{2}}-\frac{s(i, j, m, n)}{4 j^{2}} \\
= & \sum_{k=0}^{M-1} V(i, m, k) s(i-1, j, k, n) .
\end{aligned}
$$

Setting $j=i-1$ in (4.2) gives

$$
\begin{aligned}
\sum_{k=0}^{M-1} V & (i, m, k) s(i-1, i-1, k, n) \\
= & s(i-1, i-1, m, n)-s(i, i, m, n) \\
& +\frac{s(i-1, i-1, m+1, n)+s(i-1, i-1, m-1, n)-2 s(i-1, i-1, m, n)}{i^{2}} \\
& -\frac{s(i-1, i-1, m, n+1)+s(i-1, i-1, m, n-1)-2 s(i-1, i-1, m, n)}{(i-1)^{2}} .
\end{aligned}
$$


This is clearly a set of $M^{2}$ equations in the $M^{2}$ unknowns $V(i, m, k)$, since $0 \leqslant k, m, n \leqslant$ $M-1$. They can thus be solved for the $V(i, m, k)$.

The split Levinson-type layer stripping algorithm is as follows. Initialize $h(0, j, m, n)=$ 0 and $h(1, j, m, n)$ computed directly from (3.3). Propagate (3.2) in increasing $i$. For each $i$, compute $s(i, i, m, n)$ using (4.1). Compute the potential $V(i, m, n)$ by solving (4.3). Compare this with the continuous layer stripping algorithm defined at the end of section 2.3.

We call this a split Levinson-type algorithm since it consists of a multichannel recurrence that implements a discrete Schrödinger equation and requires the computation of the $s(i, i, m, n)$ using (4.1); all of these are characteristic of split Levinson-type algorithms.

The computation of the $s(i, i, m, n)$ is a major computational bottleneck since it is an inner-product-like computation. It may be avoided by using a split Schur-type algorithm, as we now demonstrate.

\subsection{Split Schur-type algorithm}

The idea of the split Schur-type algorithm is to omit the regular solution $h(i, j, m, n)$ entirely. Instead, we simply propagate $s(i, j, m, n)$ using (4.2) and recover the potential $V(i, m, n)$ by solving (4.3). This avoids the necessity of computing $s(i, j, m, n)$ from $h(i, j, m, n)$-we simply propagate the former and omit the latter entirely. The elimination of the inner-product-like computation (4.1) is characteristic of split Schur-type algorithms.

The split Schur-type layer stripping algorithm is as follows. Initialize $s(0, j, m, n)=$ $k(0, j, m, n)$. Propagate (4.2) in increasing $i$. Compute $V(i, m, n)$ by solving (4.3). Then proceed to $i+1$. This does not have a continuous layer stripping counterpart in this paper, although it is the discrete counterpart to a continuous algorithm proposed in [8].

\subsection{Split lattice-type algorithm}

In the $1 \mathrm{D}$ case the Levinson-like algorithm computes the regular solution and the Schur-like algorithm computes the scattering solution. This is not true in dimensions higher than one; the Schur variables $s(i, j, m, n)$ computed by the above algorithm are not the scattering solution $u(i, j, m, n)$ that solves (3.6).

However, we can define a layer stripping algorithm for the scattering solution. Unlike the above two algorithms, it must be initialized for large $i$ and then propagated in decreasing $i$. This makes physical sense since the scattered field is measured for $i$ outside the support of the potential. We could also start at $i=0$, but in this case computation of the potential becomes complex. Since this case is of little interest, we do not discuss it further (see [8] for a discussion of the continuous version of this algorithm). Since this algorithm is in a sense the geometric mean of the Levinson and Schur algorithms (see [8]), we call it a split lattice-type algorithm.

Analogous to defining (4.1) from (3.3), we define from (3.6)

$$
\begin{aligned}
s^{\prime}(i, j, m, n)= & \delta(i-j) \delta(m-n)+R(i, j, m, n)-u(i, j, m, n) \\
& -\sum_{k=i+1}^{\infty} \sum_{l=0}^{M-1} u(i, k, m, l) R(k, j, l, n)
\end{aligned}
$$

and by linearity $s^{\prime}(i, j, m, n)$ satisfies (3.7). Setting $j=i+1$ in (3.7) gives

$$
\begin{aligned}
& \sum_{k=0}^{M-1} V(i, m, k) s^{\prime}(i+1, i+1, k, n) \\
& \quad=s^{\prime}(i+1, i+1, m, n)-s^{\prime}(i, i, m, n)
\end{aligned}
$$




$$
\begin{aligned}
& +\frac{s^{\prime}(i+1, i+1, m+1, n)+s^{\prime}(i+1, i+1, m-1, n)-2 s^{\prime}(i+1, i+1, m, n)}{i^{2}} \\
& -\frac{s^{\prime}(i+1, i+1, m, n+1)+s^{\prime}(i+1, i+1, m, n-1)-2 s^{\prime}(i+1, i+1, m, n)}{(i+1)^{2}}
\end{aligned}
$$

since from (3.6) and (4.4) $s^{\prime}(i, j, m, n)=0$ for $j>i$. This is clearly a set of $M^{2}$ equations in the $M^{2}$ unknowns $V(i, m, k)$, since $0 \leqslant k, m, n \leqslant M-1$. They can thus be solved for the $V(i, m, k)$.

The split lattice-type layer stripping algorithm is as follows. Initialize $u(I, j, m, n)=$ $R(I, j, m, n)$ for large $I$, or directly with the scattered field $u(i, j, m, n)$ for some $i$ outside the support of the potential. Propagate (3.7) in decreasing $i$. Compute $V(i, m, n)$ by solving (4.5). Then proceed to $i-1$. Compare this with the continuous layer stripping algorithm defined in section 2.5.

\subsection{Significance of the discrete problem to continuous problem application}

The implications of our results for continuous 2D inverse scattering problems can be summarized as follows.

(1) If appropriate discretizations (3.2) and (3.3) are used, the GLM machinery for the continuous problem can be implemented exactly numerically. That is, a discrete implementation will solve a discrete problem. This shows not only that the procedure will work, but it also specifies the problem that has been solved by it.

(2) However, there are several seemingly minor but important aspects of the discrete problem that are not apparent from the continuous problem:

(a) the depth index in the angular part of the Laplacians, and in the field term multiplying the potential, must be $i \pm 1$, not $i$;

(b) the sums in the discrete GLM equations must skip every other term;

(c) the simple equation (2.33) relating the regular solution to the potential generalizes directly to (3.4). However, computation of the potential from $s(i, j, m, n)$ is not accomplished by a similar equation, but by solving the systems of equations (4.3). Note that in the continuous limit $\Delta \rightarrow 0$ the impulse in (4.1) dominates at $i=j$, so (4.3) simplifies to the simple formula

$$
\begin{aligned}
V(i+1, m, n) & =s(i, i, m, n)-s(i+1, i+1, m, n) \\
& +\frac{s(i, i, m+1, n)+s(i, i, m-1, n)-s(i, i, m, n+1)-s(i, i, m, n-1)}{i^{2}}
\end{aligned}
$$

where we have also used $i^{2} \approx(i-1)^{2}$. However, it is not at all clear how to obtain the system of equations (4.3) from the continuous-limit formula (4.6).

(3) Similar comments apply to all of the layer stripping algorithms. Note in particular that the system of equations (4.5) simplifies to (2.30) in the continuous limit $\Delta \rightarrow 0$ since again the impulse dominates.

(4) The big problem is of course nonlocality of the potential $V(i, m, n)$. If the data are miraculous, then the solution to the system of equations (4.3) will have the property $V(i, m, n)=V(i, m) \delta(m-n) ;(4.3)$ is the discrete miracle equation. But if the data are not miraculous (as they almost surely will not be), then the result of applying the GLM procedure numerically will be a nonlocal potential. The physical significance of this result, and even the interpretation of it for the continuous problem, are open to question. However, this is what actually happens numerically. 


\section{Conclusion}

We have shown that there exists a 2D discrete counterpart to the GLM machinery for the continuous 2D inverse scattering problem. The counterpart is to equations in the $x y$ domain, rather than the $x t$ domain, where the former is obtained from the latter by performing an inverse Radon transform. Since the regular solution has the property $\phi(x, t, \theta)=\phi(x,-t, \theta+\pi)$, this could be applied to the Gel'fand-Levitan procedure directly. Since the scattering solution has $\psi(x, t, \theta) \neq \psi(x,-t, \theta+\pi)$, it was necessary to define first a symmetrized $\tilde{\psi}(x, t, \theta)=\psi(x, t, \theta)+\psi(x,-t, \theta+\pi)$. Although inverse Radon transform domain equations are equivalent to the original equations only the former can be discretized exactly.

The discrete 2D inverse scattering equations include discrete Gel'fand-Levitan and Marchenko systems of equations, layer stripping algorithms that are split Levinson and Schur algorithms generalized to $2 \mathrm{D}$, and discrete counterparts to most of the continuous quantities. However, there are subtle points to the discretization that make the discrete-tocontinuous transformation easy but the continuous-to-discrete transformation much more difficult. These are summarized in section 4.3; unless these are followed there is no guarantee that discretized equations will solve a discrete problem. Appropriate discretization means that an explicitly discrete problem is being solved exactly by explicitly discrete algorithms, so the procedure will be exact for the problem it is solving. This is especially important for layer stripping algorithms, which usually diverge unless they are solving an explicitly discrete problem.

We have gone to some trouble to point out analogous equations between the discrete and continuous developments. The reader should carefully distinguish the exact discrete results from the approximations used as $\Delta \rightarrow 0$ to get the continuous results; the discrete results do not depend on $\Delta \rightarrow 0$ !

A major insight obtained by looking at the discrete problem is that almost all scattering data will be nonmiraculous, and the result of the discrete GLM procedure (whether explicitly discrete or discretized continuous) will be a nonlocal potential. This is not surprising from a degrees-of-freedom viewpoint, although its physical significance to continuous problems is open to question. However, this is what will happen when the procedure is implemented numerically.

\section{Acknowledgment}

This research was supported by the Office of Naval Research under grant no N00014-94$1-0519$.

\section{References}

[1] Newton R G 1980 Inverse scattering. II. Three dimensions J. Math. Phys. 21 1698-715

[2] Newton R G 1981 Inverse scattering III. Three dimensions, continued J. Math. Phys. 22 2191-200

[3] Newton R G 1982 Inverse scattering IV. Three dimensions: generalized Marchenko construction with bound states, and generalized Gel'fand-Levitan equations J. Math. Phys. 23 594-604

[4] Rose J H, Cheney M and DeFacio B 1984 The connection between time and frequency domain threedimensional inverse scattering methods J. Math. Phys. 25 2995-3000

[5] Yagle A E 1987 Multi-dimensional inverse scattering: an orthogonalization formulation J. Math. Phys. 28 1481-91

[6] Cheney M 1984 Inverse scattering in dimension two J. Math. Phys. 25 94-102 
[7] Yagle A E 1988 Differential and integral methods for three-dimensional inverse scattering problems with a non-local potential Inverse Problems 4 549-66

[8] Yagle A E 1990 Analogues of split Levinson, Schur, and lattice algorithms for three-dimensional random field estimation problems SIAM J. Appl. Math. 50 1780-99

[9] Kay I and Moses H E 1961 A simple verification of the Gelfand-Levitan equation for the three-dimensional scattering problem Comment. Pure Appl. Math. 14 435-45

[10] Kay I and Moses H E 1961 The determination of the scattering potential from the spectral measure function V. The Gel'fand-Levitan equation for the three dimensional scattering problem Nuovo Cimento 22 689-705

[11] Bruckstein A M and Kailath T 1987 Inverse scattering for discrete transmission-line models SIAM Rev. 29 359-89

[12] Bruckstein A M, Levy B C and Kailath T 1985 Differential methods in inverse scattering SIAM J. Appl. Math. 45 312-35

[13] Yagle A E and Levy B C 1986 Layer stripping solutions of multi-dimensional inverse scattering problems $J$. Math. Phys. 27 1701-10

[14] Yagle A E and Raadhakrishnan P 1992 Numerical performance of layer stripping algorithms for 2D inverse scattering problems Inverse Problems 8 645-65

[15] Yagle A E and Frolik J L 1996 On the feasibility of impulse reflection response data for the 2D inverse scattering problem IEEE Trans. Antennas Propag. 44 1551-64

[16] Yagle A E 1988 Connections between three-dimensional inverse scattering and the linear least-squares estimation of random fields Acta Appl. Math. 13 267-89

[17] Fang W-H and Yagle A E 1992 Discrete fast algorithms for 2D linear prediction on a polar raster IEEE Trans. Signal Process. 40 1480-9

[18] Robinson E 1975 Dynamic predictive deconvolution Geophys. Prospect. 23 779-97

[19] Chadan K and Sabatier P 1989 Inverse Problems in Quantum Scattering Theory 2nd edn (New York: Springer)

[20] Newton R G 1989 Inverse Schrödinger Scattering in Three Dimensions (New York: Springer)

[21] Weder R 1991 Characterization of the scattering data in multidimensional inverse scattering theory Inverse Problems 7 461-89

[22] Tricomi F G 1957 Integral Equations (New York: Interscience)

[23] Eskin G and Ralston J 1992 Inverse backscattering J. Analyse Math. 58 177-90 